\title{
Flow Induced in Fluid-Particle Suspension by an Infinite Rotating Disk*
}

\author{
LAURence B. Zung† \\ Daniel and Florence Guggenheim Jet Propulsion Center, Karman Laboratory of Fluid Mechanics and Jet Propulsion. \\ California Institute of Technology, Pasadena, California \\ (Received 18 March 1968; final manuscript received 21 October 1968)
}

\begin{abstract}
An exact similarity solution is given for the flow of a fluid-particle suspension over an infinitely large disk rotating at a constant velocity. Numerical solutions of the resulting ordinary differential equations provide velocity distributions for both fluid and solid phases and density distributions for the solid. The boundary-layer thicknesses of the particle cloud and the fluid are found to be approximately equal. In addition to its intrinsic value as a solution to a physical problem, the results provide a convenient basis for judging the accuracy of approximate techniques.
\end{abstract}

\section{INTRODUCTION}

Because of the complexity of fluid-particle flow problems, most analyses in this field involve perturbation schemes ${ }^{1,2}$ or approximation methods. ${ }^{3}$ As a consequence, exact solutions of problems in twophase flow take on special interest.

One example of such exact solutions is the flow over an infinitely large disk rotating at a constant velocity about an axis perpendicular to its plane. Von Kármán ${ }^{4}$ first used the integral method to investigate a similar problem of an incompressible viscous fluid. Using numerical integration, an exact solution was obtained by Cochran. ${ }^{5}$

The layer of two-phase medium close to the rotating disk is carried along by the disk. Owing to the centrifugal forces, this layer is being transported outwardly towards the outer edges. This, in turn, is compensated for by the flow along the axial direction. Thus, the axial velocities of the fluid and the particle cloud do not vanish far away from the disk, but tend to a constant value. On the surface of the disk, the radial velocity of the fluid vanishes, while the radial velocity of the particle cloud usually attains its maximum value. The flow field exhibits very well the centrifugal separation of particulate matter from fluid as well as the characteristics of a secondary flow of a twophase medium in a three-dimensional boundary layer.

* This work constitutes Chapter I of the author's Ph.D. thesis, California Institute of Technology (1967).

$\dagger$ Present address: Dynamic Science, Monrovia, California.

1 F. E. Marble, in Proceedings of the Fifth AGARD Combustion and Propulsion Colloquium (Pergamon Press, Ltd. London, 1963), p. 192.

2 F. E. Marble, AIAA J. 1, 2793 (1963).

3. R. Kliegel, in Ninth Symposium (International) on Combustion (Academic Press Inc., New York, 1963), p. 811.

4 Th. von Kármán, Z. Angew. Math. Mech. 1, 233 (1921).

6 G. W. Cochran, Proc. Cambridge Phil. Soc. 30, 365,

\section{BASIC EQUATIONS}

A half-infinite space, $z>0$, is filled with an incompressible fluid containing small particles of a single size with radius $\sigma$. The boundary plane, $z=0$, is rotating at a constant angular velocity $\omega$. Using cylindrical coordinates and taking account of the rotational symmetry, we easily observe that the flow quantities are independent of the angle $\theta$. The governing equations describing the flow field of a two-phase medium are discussed in detail in Ref. 1. Denote the radial, angular, and axial velocities of the fluid by $u, v$, and $w$; the mass density by $\rho$; and designate quantities associated with the particle cloud by subscript $p$. The continuity equations for the two phases are

$$
\begin{gathered}
\frac{\partial}{\partial r}(r u)+\frac{\partial}{\partial z}(r w)=0, \\
\frac{\partial}{\partial r}\left(r \rho_{p} u_{p}\right)+\frac{\partial}{\partial z}\left(r \rho_{p} w_{p}\right)=0 .
\end{gathered}
$$

The corresponding momentum equations are

$$
\begin{aligned}
& u \frac{\partial u}{\partial r}-\frac{v^{2}}{r}+w \frac{\partial u}{\partial z} \\
& =-\frac{1}{\rho} \frac{\partial p}{\partial r}+\nu\left(\frac{\partial^{2} u}{\partial r^{2}}+\frac{\partial}{\partial r} \frac{u}{r}+\frac{\partial^{2} u}{\partial z^{2}}\right)+\frac{1}{\rho} F_{r} \\
& u \frac{\partial v}{\partial r}+\frac{u v}{r}+w \frac{\partial v}{\partial z} \\
& =\nu\left(\frac{\partial^{2} v}{\partial r^{2}}+\frac{\partial}{\partial r} \frac{v}{r}+\frac{\partial^{2} v}{\partial z^{2}}\right)+\frac{1}{\rho} F_{\theta}
\end{aligned}
$$

$$
\begin{aligned}
& u \frac{\partial w}{\partial r}+w \frac{\partial w}{\partial z} \\
& =-\frac{1}{\rho} \frac{\partial p}{\partial z}+\nu\left(\frac{\partial^{2} w}{\partial r^{2}}+\frac{1}{r} \frac{\partial w}{\partial r}+\frac{\partial^{2} w}{\partial z^{2}}\right)+\frac{1}{\rho} F_{z}
\end{aligned}
$$




$$
\begin{aligned}
& u_{p} \frac{\partial u_{p}}{\partial r}-\frac{v_{p}^{2}}{r}+w_{p} \frac{\partial u_{p}}{\partial z}=-\frac{1}{\rho_{p}} F_{r}, \\
& u_{p} \frac{\partial v_{p}}{\partial r}+\frac{u_{p} v_{p}}{r}+w_{p} \frac{\partial v_{p}}{\partial z}=-\frac{1}{\rho_{p}} F_{\theta}, \\
& u_{p} \frac{\partial w_{p}}{\partial r}+w_{p} \frac{\partial w_{p}}{\partial z}=-\frac{1}{\rho_{p}} F_{z},
\end{aligned}
$$

where $p$ is the local gas pressure and $F_{r}, F_{\theta}$, and $F_{z}$ are the forces in the radial, angular, and axial directions exerted on a unit volume of fluid by the particles.

Using a linear drag law as a first approximation to describe the particle-fluid interaction $F_{r}$ is

$$
F_{r}=\tau^{-1} \rho_{p}\left(u_{p}-u\right),
$$

where $\tau$ has the dimension of time. In the case when Stokes drag law is a valid approximation, $\tau$ assumes a simple form

$$
\tau=m / 6 \pi \mu \sigma,
$$

where $m$ is the mass of a single particle. Similarly, $F_{\theta}$ and $F_{z}$ are

$$
\begin{aligned}
& F_{\theta}=\tau^{-1} \rho_{p}\left(v_{p}-v\right), \\
& F_{z}=\tau^{-1} \rho_{p}\left(w_{p}-w\right) .
\end{aligned}
$$

The boundary conditions for the fluid properties are

$$
\begin{array}{llll}
u=0, & v=r \omega, & w=0 & \text { for } z=0, \\
u=0, & v=0, & & \text { for } z=\infty, \\
p=p_{\infty} . & & &
\end{array}
$$

As was mentioned previously $w$ does not vanish as $z \rightarrow \infty$, but tends towards a constant value. The boundary conditions for the quantities pertaining to the particle cloud are

$$
\begin{aligned}
& u_{p}=0, \quad v_{p}=0, \quad w_{p}=w \text { for } z=\infty \text {, } \\
& \rho_{p}=\kappa \rho \quad \text { for } z=\infty \text {, }
\end{aligned}
$$

where $k$ is the density ratio for $z=\infty$ and is equal to a constant.

\section{SOLUTION FOR THE EQUILIBRIUM FLOW}

From the definition of $\tau$, Eq. (10), the limiting case when $\tau \rightarrow 0$ corresponds to either very small particles or the viscosity of the fluid $\mu$ is very large. Under such circumstances, the velocity difference between the particle cloud and the fluid is expected to be very small. This is the case when we examine Eqs. (6), (7), and (8). Define

$$
\begin{aligned}
& u_{s}=u_{p}-u, \\
& v_{s}=v_{p}-v, \\
& w_{s}=w_{p}-w .
\end{aligned}
$$

The momentum equations for the particle cloud can be expressed as

$$
\begin{aligned}
& u \frac{\partial u}{\partial r}-\frac{v^{2}}{r}+w \frac{\partial u}{\partial z}=-\frac{u_{s}}{\tau}+O\left(u_{s}\right) \\
& u \frac{\partial v}{\partial r}+\frac{u v}{r}+w \frac{\partial v}{\partial z}=-\frac{v_{s}}{\tau}+O\left(v_{s}\right) \\
& u \frac{\partial w}{\partial r}+w \frac{\partial w}{\partial z}=-\frac{w_{s}}{\tau}+O\left(w_{s}\right)
\end{aligned}
$$

The continuity equation is

$$
\frac{\partial}{\partial r}\left(r u \rho_{p}\right)+\frac{\partial}{\partial z}\left(r w \rho_{p}\right)=O\left(u_{s}, w_{s}\right) .
$$

Since both the velocities and their spatial derivatives are finite, the limiting case when $\tau \rightarrow 0$ requires $u_{*} \rightarrow 0, v_{s} \rightarrow 0$, and $w_{s} \rightarrow 0$. Thus, Eqs. (16)-(19) are reduced to

$$
\begin{aligned}
& u \frac{\partial u}{\partial r}-\frac{v^{2}}{r}+w \frac{\partial u}{\partial z}=-\frac{u_{s}}{\tau}=-\frac{u_{p}-u}{\tau} \\
& u \frac{\partial v}{\partial r}+\frac{u v}{r}+w \frac{\partial v}{\partial r}=-\frac{v_{s}}{\tau}=-\frac{v_{p}-v}{\tau} \\
& u \frac{\partial w}{\partial r}+w \frac{\partial w}{\partial z}=-\frac{w_{s}}{\tau}=-\frac{w_{p}-w}{\tau} .
\end{aligned}
$$

From the continuity equation we observe that $\rho_{p}=$ const $=\kappa \rho$.

The momentum equations for the whole system, in the limiting case when $\tau \rightarrow 0$, can be expressed as $u \frac{\partial u}{\partial r}-\frac{v^{2}}{r}+w \frac{\partial u}{\partial z}$

$$
\begin{aligned}
& =-\frac{1}{\rho^{*}} \frac{\partial p}{\partial r}+\nu^{*}\left(\frac{\partial^{2} u}{\partial r^{2}}+\frac{\partial}{\partial r} \frac{u}{r}+\frac{\partial^{2} u}{\partial z^{2}}\right) \\
u \frac{\partial v}{\partial r}+ & \frac{u v}{r^{2}}+w \frac{\partial v}{\partial z}=\nu^{*}\left(\frac{\partial^{2} v}{\partial z^{2}}+\frac{\partial}{\partial r} \frac{v}{r}+\frac{\partial^{2} v}{\partial z^{2}}\right) \\
u \frac{\partial w}{\partial r}+ & w \frac{\partial w}{\partial z} \\
= & -\frac{1}{\rho^{*}} \frac{\partial p}{\partial z}+\nu^{*}\left(\frac{\partial^{2} w}{\partial z^{2}}+\frac{1}{r} \frac{\partial w}{\partial r}+\frac{\partial^{2} w}{\partial z^{2}}\right)
\end{aligned}
$$

where

$$
\begin{aligned}
& \rho^{*}=\rho+\rho_{p}(\infty)=\rho(1+\kappa), \\
& \nu^{*}=\mu /\left[\rho+\rho_{p}(\infty)\right]=\mu /(1+\kappa) \rho .
\end{aligned}
$$


The solution for $\tau \rightarrow 0$ is, therefore, identical with the flow without particles with an effective density $\rho^{*}=\rho(1+\kappa)$. This limiting case is usually referred to as the "equilibrium flow."

In another limiting case when $r_{v} \rightarrow \infty$, the presence of the particle cloud does not change the fluid quantities. The particles are moving with constant velocities $u_{p}=0, v_{p}=0, w_{D}(r, \infty)$, and $\rho_{p}=\kappa \rho$. This case is usually referred to as the "frozen flow."

\section{METHOD OF SOLUTION}

To investigate the problem for finite values of $\tau$, it is convenient to introduce a dimensionless distance variable $\eta . \eta$ is defined as

$$
\eta=(\omega / \nu)^{\frac{1}{2}} z .
$$

By expressing the fluid velocity components and pressure in the following form:

$$
\begin{aligned}
u(r, z) & =r \omega F(\eta), \\
v(r, z) & =r \omega G(\eta), \\
w(r, z) & =(\omega \nu)^{\frac{1}{2}} H(\eta), \\
p(r, z) & =p(r, \infty)=\rho \omega \nu P(\eta),
\end{aligned}
$$

defining a relative density $\rho_{r}=\rho_{p} / \rho$, and expressing the particle velocities and density as

$$
\begin{aligned}
u_{p}(r, z) & =\rho_{r}^{-1} r \omega F_{p}(\eta), \\
v_{p}(r, z) & =\rho_{r}^{-1} r \omega G_{p}(\eta), \\
w_{p}(r, z) & =\rho_{r}^{-1}(\nu \omega)^{\frac{1}{2}} H_{p}(\eta), \\
\rho_{r}(r, z) & =Q(\eta),
\end{aligned}
$$

the governing equations (1)--(8) are reduced to the following form:

$$
\begin{aligned}
& \frac{d H}{d \eta}+2 F=0 \\
& \frac{d^{2} F}{d \eta^{2}}-F^{2}-H \frac{d F}{d \eta}+G^{2}+\beta\left(F_{p}-Q F^{\prime}\right)=0 \\
& \frac{d^{2} G}{d \eta^{2}}-2 F G-H \frac{d G}{d \eta}+\beta\left(G_{p}-Q G\right)=0 \\
& \frac{d P}{d \eta}-2 H F+2 \frac{d F}{d \eta}+\beta\left(-H_{p}+Q H\right)=0 \\
& \frac{d H_{p}}{d \eta}+2 F_{p}=0, \quad-\beta\left(Q^{2} F_{p}-Q^{3} F\right)=0 \\
& -H_{p} Q \frac{d F_{p}}{d \eta}+H_{p} \frac{d Q}{d \eta} F_{p}+G_{p}^{2} Q-Q F_{p}^{2}
\end{aligned}
$$

$$
\begin{array}{r}
H_{p} Q \frac{d G_{p}}{d \eta}-2 Q G_{p} F_{p}+H_{p} G_{p} \frac{d Q}{d \eta} \\
-\beta\left(Q^{2} G_{p}-Q^{3} G\right)=0, \\
H_{p}^{2} \frac{d Q}{d \eta}+2 H_{p} Q F_{p}-\beta\left(Q^{2} H_{p}-Q^{3} H\right)=0 .
\end{array}
$$

The boundary conditions can be obtained from Eqs. (13) and (14). They are

$$
\begin{gathered}
F(\eta)=0, \quad G(\eta)=1, \quad H(\eta)=0 \text { for } \eta=0, \\
F(\eta)=0, \quad G(\eta)=0, \quad P(\eta)=0 \text { for } \eta=\infty, \\
F_{p}(\eta)=0, G_{p}(\eta)=0, H_{p}(\eta)=\kappa H(\eta) \\
\text { for } \eta=\infty, \\
Q(\eta)=\kappa \text { for } \eta=\infty .
\end{gathered}
$$

$\beta$ is defined as

$$
\beta=1 / \omega \tau .
$$

Asymptotic solutions which are valid for large values of $\eta$ were first obtained. Functions $F(\eta)$, $F_{p}(\eta)$, etc., were evaluated for some large value of $\eta=\eta_{0}$ using the asymptotic solutions. Numerical integration was carried out from the point $\eta_{0}$ to $\eta=0$. The asymptotic solutions contain three parameters. Using an iteration procedure based on Newton's method, the boundary conditions on the disk, namely, $F(0)=0, G(0)=1$, and $H(0)=0$, were satisfied by varying the three parameters. Numerical integration was performed with an IBM 7094 computer using the DEQ subroutine.

Two sets of solutions were obtained by varying, respectively, the particle loading $\kappa$ and the particlefluid interaction parameter $\beta$.

\section{DISCUSSION}

The fluid velocity profiles are shown in Figs. 1 and 2 for various particle loading $\kappa$. Figures 3 and 4 show the corresponding particle velocity profiles. Several observations can be made based on these solutions. The flow field is very much like Prandtl's boundary-layer type. The boundary-layer thicknesses $\delta$ and $\delta_{p}$ for the fluid and the particle cloud are approximately equal. The distances from the wall over which the angular velocities of the particle cloud and the fluid are reduced to half of their disk values differ by less than $10 \%$. Their values are close to $(\nu / \omega)^{\frac{1}{2}}$. For small values of $\delta$, the radial and angular velocities of both phases have appreciable values only within a layer of thickness of the order $(\nu / \omega)^{\frac{1}{2}}$. Close to the disk, $u_{p}$ usually attains its maximum value while $u$ approaches zero. This is 


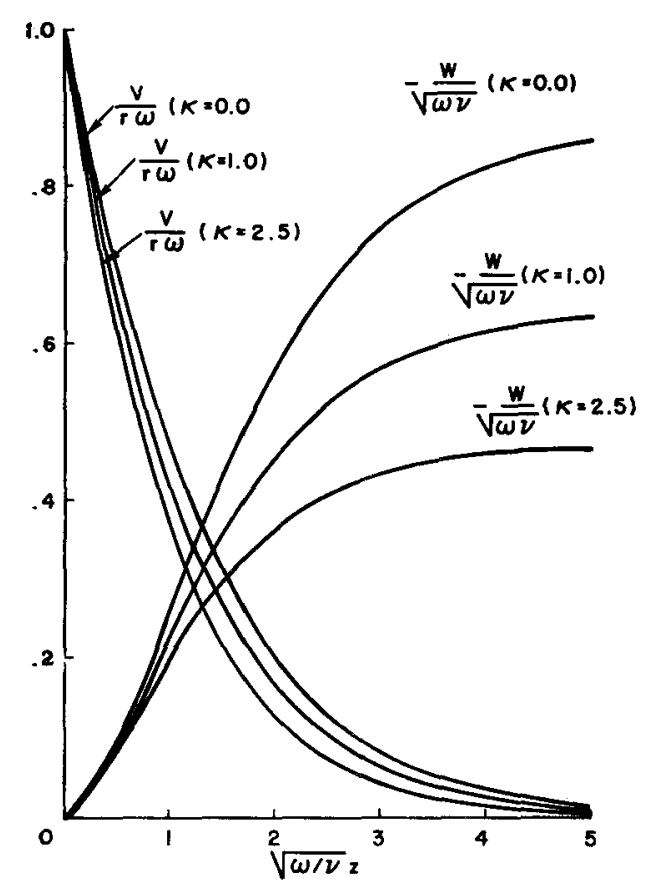

FIG. 1. Dependence of fluid velocities in tangential and axial directions upon particle loading $(\beta=0.5)$.

simply because of the large centrifugal force exerted on the particle cloud. The value of $u_{p}$ very close to the disk depends on the interaction parameter $\beta$. Figures 5 and 6 show the particle velocity profiles for various particle-fluid interaction parameters $\beta$. For $\beta$ equal to 0.2 , the nondimensionalized velocity $u_{p} /(\omega r)$ has a value of 0.12 , while for $\beta$ equal to 1.0, $u_{p} /(\omega r)$ is close to 0.3. This is attributed to the difference in angular velocity of the particle cloud; $v_{x} /(\omega r)$ is 0.24 and 0.62 , respectively, for $\beta$ equal to 0.2 and 1.0. For large values of $\beta, u_{p}$ on the wall

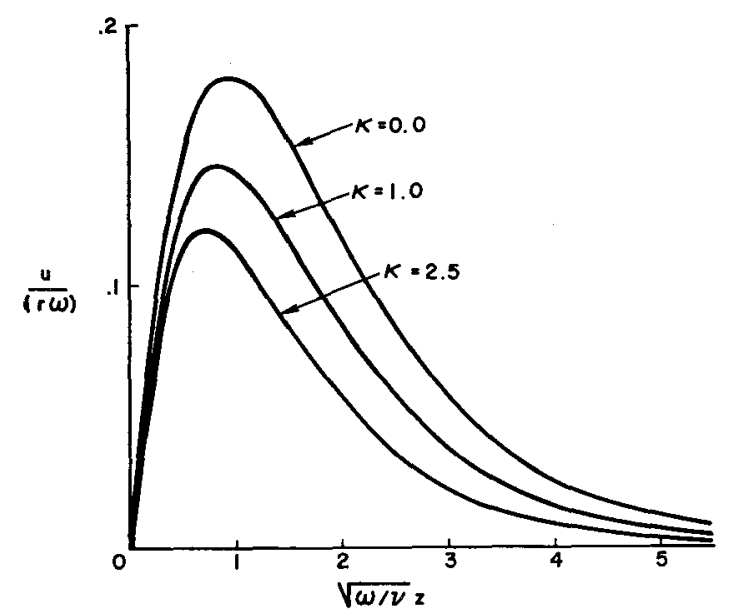

FrG. 2. Dependence of fluid velocities in radial directions upon particle loading $(\beta=0.5)$.

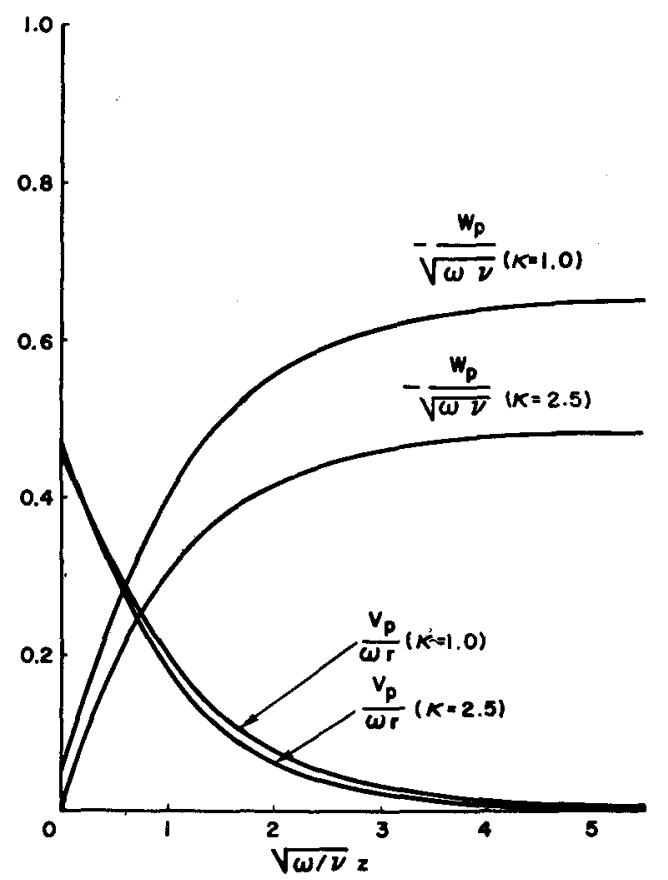

FIG. 3. Dependence of particle velocities in tangential and axial directions upon particle loading $(\beta=0.5)$.

again decreases, and for infinitely large values of $\beta$ when there is equilibrium flow, $u_{p}$ approaches zero on the wall.

The change of particle density and fluid pressure for various particle loading $\kappa$ is shown in Figs. 7

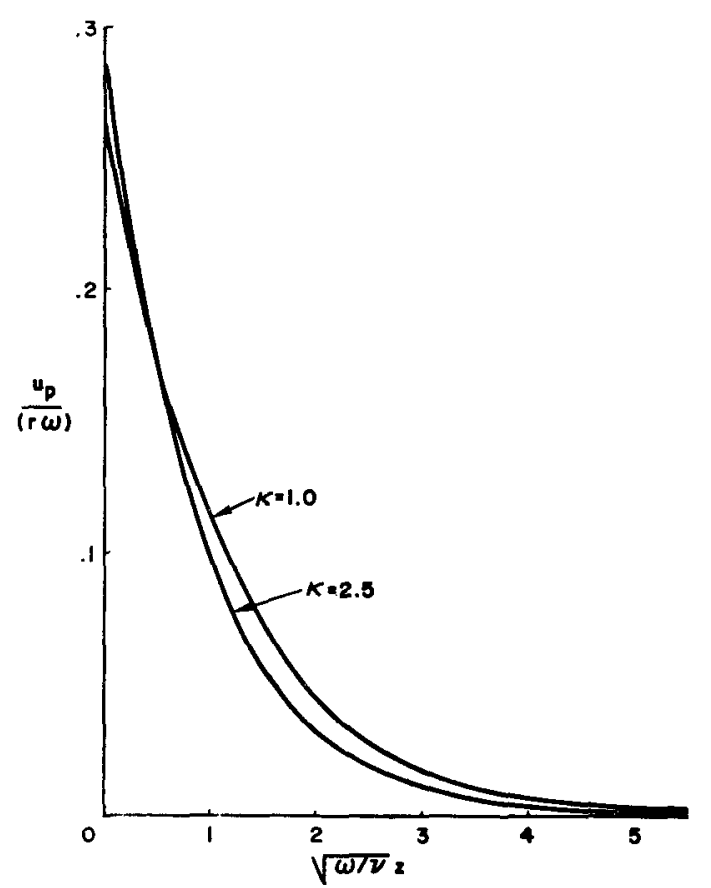

FIG. 4. Dependence of particle velocities in radial direction upon particle loading ( $\beta=0.5$ ). 


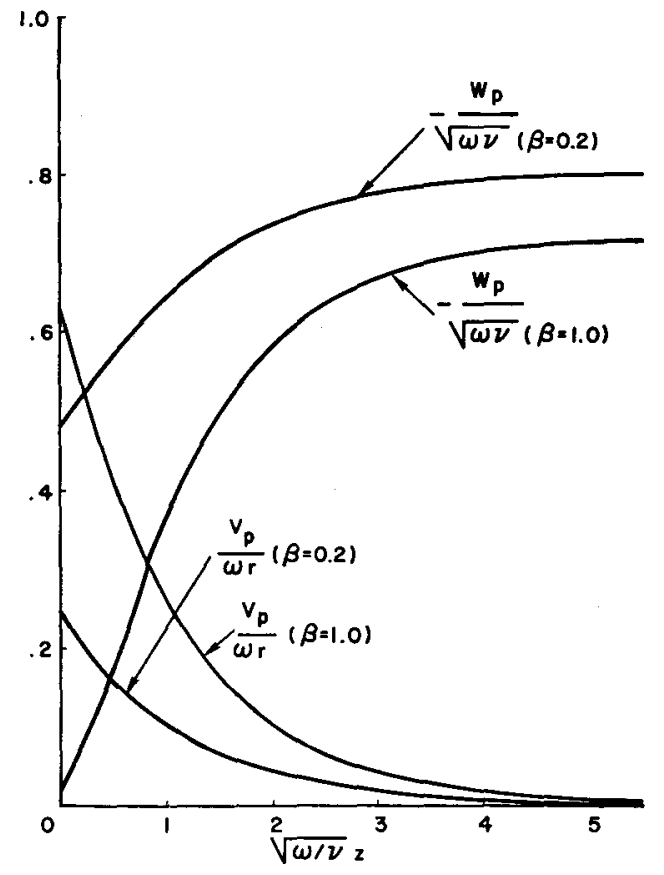

FIG. 5. Dependence of particle velocity in tangential and axial directions upon interaction parameter $\beta(\kappa=0.5)$.

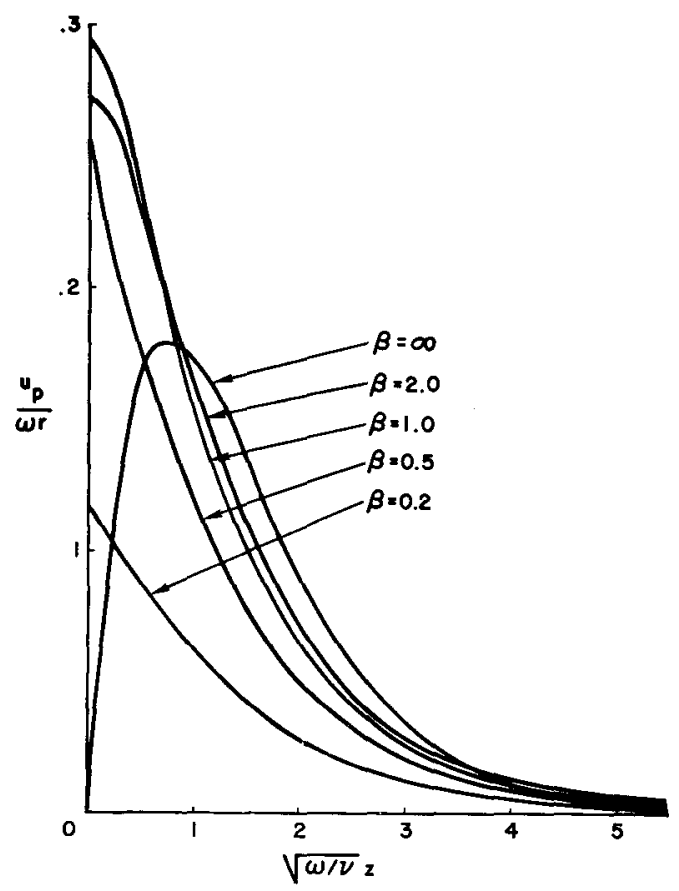

FIG. 6. Dependence of particle velocity in radial direction upon interaction parameter $\beta(x=0.5)$.

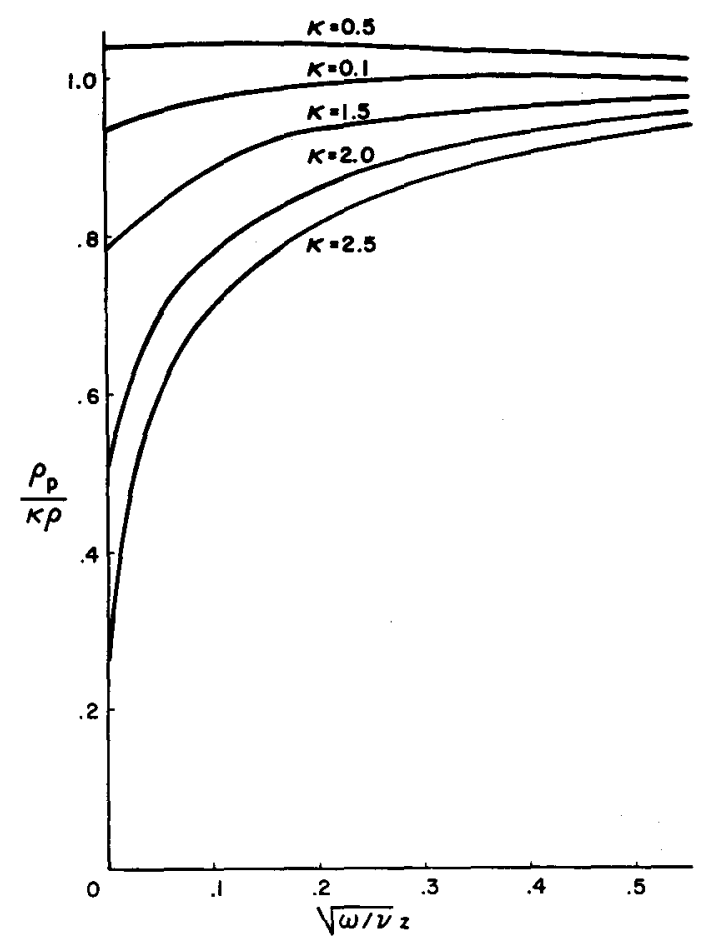

FIG. 7. Dependence of particle density upon particle loading $(\beta=0.5)$.

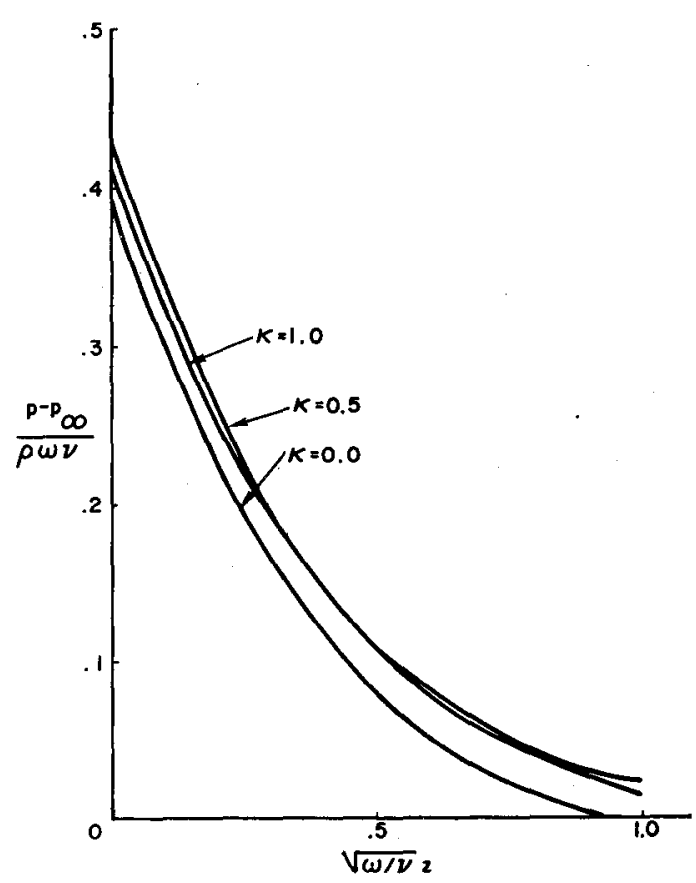

FIG. 8. Dependence of pressure upon particle loading $(\beta=0.5)$. 


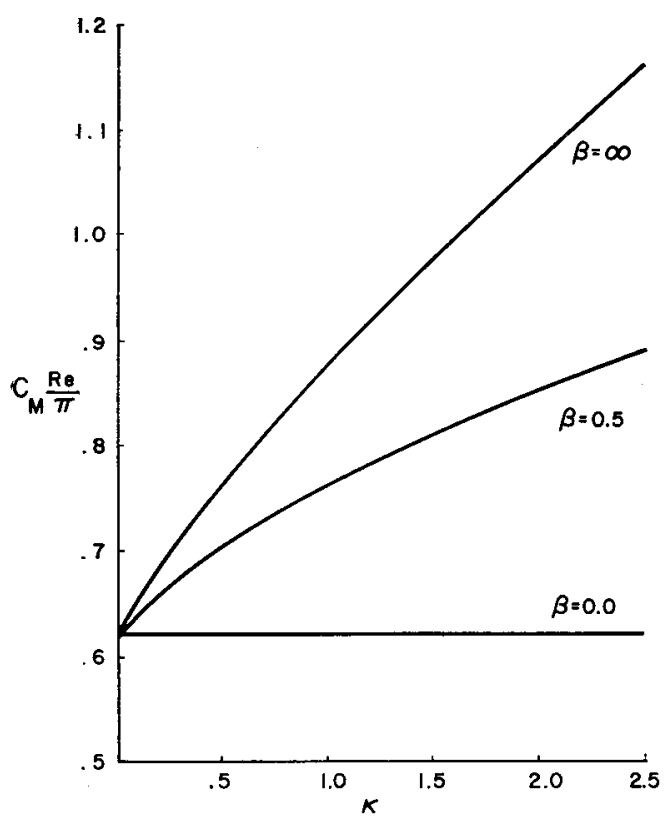

Frg. 9. Dependence of coefficient of turning moment upon particle loading for different interaction parameter $\beta$.

and 8 . The pressure attains its maximum value on the disk. The two phase mixture is thus flowing in a region with an adverse pressure gradient. This is contrary to the solution obtained by Cochran, ${ }^{5}$ since he has a sign error in the pressure distribution.

It is noteworthy that $w_{p}$ does not vanish on the disk. The problem is solved with the boundary condition that the particles stick to the disk or that the amount of particles reflected from the disk is negligible. This latter assumption is well justified, since from the solution we observe that $w_{p}$ on the disk is indeed small except for very small values of $\beta$. Furthermore, the axial velocities are of the order $(\nu \omega)^{\frac{1}{2}}$ and at any rate are small.

When the radius $R$ of the disk is large compared with the boundary-layer thickness, the boundary conditions along the edge of the disk can be neglected. The solution for the infinite disk can be used in this case to evaluate the turning moment

$$
M=\int_{0}^{R} 2 \pi r^{2} \tau_{\theta z} d r
$$

of the disk. Define $C_{M}=M /\left(\frac{1}{2} \rho \omega^{2} R^{5}\right)$ as the coefficient of the turning moment of the disk; $C_{M}$ can be easily calculated and is equal to

$$
C_{M}=-\left.\frac{d G}{d \eta}\right|_{\eta=0} / \operatorname{Re}
$$

where $R e$ is the Reynolds number defined as $\operatorname{Re}=$ $R^{2} \omega / \nu . C_{M}$ increases for increasing values of $\beta$ and $\kappa$ as shown in Fig. 9.

\section{ACKNOWLEDGMENTS}

The author is indebted to Professor Frank E. Marble for his guidance and helpful suggestions.

This work was supported by Aerospace Research Laboratories, Office of Aerospace Research, under Contract AF33(615)-3785. 